\title{
Assessment of radioiodine therapy efficacy for treatment of differentiated thyroid cancer patients with pulmonary metastasis undetected by chest computed tomography
}

\author{
BIN LONG, MENGDI YANG, ZHIWEN YANG, HEQING YI and LINFA LI \\ Department of Nuclear Medicine, Zhejiang Cancer Hospital, Hangzhou, Zhejiang 310022, P.R. China
}

Received December 15, 2014; Accepted November 18, 2015

DOI: $10.3892 / \mathrm{ol} .2015 .4034$

\begin{abstract}
Radioiodine therapy (RAI) has proven effective for the treatment of patients exhibiting differentiated thyroid cancer (DTC) with pulmonary metastases. However, the early detection of metastasis remains challenging, and various studies have reported variations in radioiodine treatment efficacy. The present study investigated whether RAI is an effective method for the treatment of DTC with pulmonary metastases undetected by computed tomography (CT). A retrospective study was performed, analyzing iodine-131 $\left({ }^{131} \mathrm{I}\right)$ therapy in $21 \mathrm{DTC}$ patients with lung metastases that were undetected by CT. All 21 patients were initially treated with radioiodine ablation of thyroid remnants. Routine chest CT was performed prior to ${ }^{131} \mathrm{I}$ treatment without diagnostic radioiodine whole-body scanning (DxWBS), and post-therapeutic WBS was performed 3-5 days subsequent to oral administration of ${ }^{131} \mathrm{I}$. The overall effectiveness rate was $95.2 \%(20 / 21)$. The rates for complete response (CR), partial response and no response were 23.8 (5/21), $71.4(15 / 21)$ and $4.8 \%(1 / 21)$, respectively. There were 12 patients with diffuse uptake, and the remaining 9 patients demonstrated focused and low uptake. The difference in CR rate between diffuse uptake and focused uptake patients was not statistically significant $(\mathrm{P}=0.123)$. A correlation was observed between thyroglobulin (Tg) levels and extrapulmonary metastases. All patients exhibited extrapulmonary metastases when Tg levels were $>87.5 \mathrm{ng} / \mathrm{ml}$ (area under receiver operating characteristic curve, $1.0 ; \mathrm{P}<0.001)$. Overall, DTC patients with lung metastases undetected by $\mathrm{CT}$ imaging responded well to ${ }^{131} \mathrm{I}$ radiotherapy and demonstrated a positive prognosis. Serum Tg levels prior to ${ }^{131} \mathrm{I}$ treatment may correlate with metastasis, and this may suggest a requirement for the performance of DxWBS prior to radiotherapy.
\end{abstract}

Correspondence to: Dr Linfa Li, Department of Nuclear Medicine, Zhejiang Cancer Hospital, 38 Guangji Road, Hangzhou, Zhejiang 310022, P.R. China

E-mail: lilinfazj@outlook.com

Key words: differentiated thyroid cancer, pulmonary metastasis, radioiodine

\section{Introduction}

Differentiated thyroid cancer (DTC) is one of the most commonly observed types of endocrine cancer, and includes papillary TC (PTC) and follicular TC (FTC) (1). TC incidence rates have increased significantly in recent years for various reasons (1). In general, the prognosis of DTC is positive, however, patients exhibiting distant metastases at the time of diagnosis demonstrate a markedly worse prognosis (1). The lungs are the most frequent distant metastatic sites, with an incidence rate of $2-20 \%$ (2). The treatment options for adult DTC include surgery, radioiodine therapy (RAI) and thyroid hormone suppression, while long-term monitoring is essential following treatment (3). The current treatment plan is based on total or near-total thyroidectomy, followed by radioiodine remnant ablation and subsequent hormone replacement therapy (3). Measurement of serum thyroglobulin ( $\mathrm{Tg}$ ) levels, ultrasonography or single-photon emission computed tomography (SPECT)/CT and radioiodine diagnostic whole-body scanning (DxWBS) are generally performed during follow-up examination (4). The majority of patients exhibiting lung metastases are diagnosed using chest $\mathrm{CT}$ and ${ }^{131} \mathrm{I}-\mathrm{WBS}$ prior to RAI $(2,4)$. DxWBS is frequently performed for the early detection of metastases, however, it remains controversial whether DxWBS is sensitive enough to detect early pulmonary metastases, and whether treatment dosage is affected due to its effect of reducing ${ }^{131} \mathrm{I}$ uptake (5). By contrast, increased $\mathrm{Tg}$ levels following thyroidectomy and radioiodine remnant ablation indicate the persistence or recurrence of an active tumor $(6,7)$. However, there are patients who produce negative CT scans and ${ }^{131} \mathrm{I}-\mathrm{WBS}$ positivity for metastases, and it remains to be elucidated whether RAI is effective in these patients. Another issue to be elucidated is whether increased Tg levels are a prerequisite for the performance of DxWBS. The present study is a retrospective report analyzing ${ }^{131} \mathrm{I}$ therapy in 21 DTC patients exhibiting lung metastases that were undetected by CT.

\section{Materials and methods}

Clinical data. A total of 3,802 patients received RAI for the treatment of DTC in the Department of Nuclear Medicine at Zhejiang Cancer Hospital (Hangzhou, China) between January 2007 and September 2012. Among these patients, 419 exhibited lung metastases, identified by clinical 
examination, imaging and laboratory examination. A total of 21 patients demonstrated no evidence of lung metastases, as revealed by chest $\mathrm{CT}$ prior to the initial administration of RAI, and these patients exhibited abnormal lung iodine uptake in post-therapeutic WBS (RxWBS). Among these 21 patients, 11 were male and 10 were female, and the median age was $35.4 \pm 13.5$ years (range, $17-59$ years). All patients were treated with a near-total or total thyroidectomy, and the pathological tumor classification was PTC (8). The patients exhibited no lung disease or secondary malignant tumors prior to iodine treatment, and follow-up times ranged between 18 and 84 months (median, 36 months).

Treatment plan. All 21 DTC patients who exhibited lung metastases undetected by chest CT scans were initially treated with radioiodine ablation of thyroid remnants, and subsequent pretreatment required an iodine-free diet and thyroid hormone withdrawal for 3-4 weeks. All patients exhibited a clinical hypothyroid state, with serum thyroid-stimulating hormone levels $(\mathrm{TSH})$ of $>30 \mathrm{mU} / \mathrm{l}$ (normal range, 0.38-4.34 mU/1). Routine chest CT was performed prior to ${ }^{131} \mathrm{I}$ treatment without DxWBS, for which the dosage was 3.7 GBq. RxWBS was performed 3-5 days after the oral administration of ${ }^{131} \mathrm{I}$, with SPECT/CT fusion imaging if required. The interval time between rounds of repeated ${ }^{131}$ I treatment was 4-6 months, and the single treatment dosage was 5.55-7.4 GBq.

Instruments and materials. An ${ }^{131} \mathrm{I}$ solution was purchased from Chengdu Zhonghe Radioisotope, Inc., (Chengdu, China) and ${ }^{131} \mathrm{I}$ imaging was performed using an Infinia Hawkeye 4 (GE Healthcare Bio-Sciences, Pittsburgh, PA, USA) with SPECT/CT. Chest CT was independently performed by the radiologists/technicians from the Department of Radiotherapy in Zhejiang Cancer Hospital. Serum Tg and Tg-antibody blood tests were performed using chemiluminescence (Kit $\mathrm{Tg}$, cat no. 16909903; Kit Tg Ab, cat no. 16826601; Roche Diagnostics, Ltd., Burgess Hill, UK), with a normal range of 1.4-78 $\mathrm{ng} / \mathrm{ml}$ and 0-115 U/ml, respectively.

Diagnosis of DTC lung metastasis undetected by chest CT scan. A lung ${ }^{131} \mathrm{I}$ uptake value higher than the normal basal level, excluding the physiological uptake and contamination from the body surface, was considered to be RxWBS-positive. Lung CT (with 5-mm scanning depth) results demonstrating no evident bulky nodules or other abnormal high intensity bulk were considered negative. RxWBS and chest CT results were independently assessed by two doctors/radiologists from the Department of Nuclear Medicine and the Department of Radiology (Zhejiang Cancer Hospital), respectively.

Assessment of therapeutic effectiveness. The following criteria were used to assess therapeutic effectiveness: i) Complete response (CR), no clinical symptoms of lung metastases, no abnormal lung uptake in ${ }^{131} \mathrm{I}-\mathrm{WBS}$ and other imaging examinations, and Tg negativity (serum Tg levels $<1 \mathrm{ng} / \mathrm{ml}$ with TSH stimulation); ii) partial response (PR), lung uptake reduced in ${ }^{131}$ I-WBS and negative lung metastases in other imaging examinations, with decreased $\mathrm{Tg}$ levels (compared with either TSH inhibition or stimulation); iii) no response (NR), no improvement or no change in lung uptake or other imaging examinations, with lung metastases in other imaging examinations and increased Tg levels. CR and PR were considered to indicate effective ${ }^{131} \mathrm{I}$ therapy.

Statistical analysis. Data were analyzed using SPSS (version 19.0; IBM SPSS, Armonk, NY, USA), and the quantitative data are presented as the mean \pm standard deviation. Distribution was analyzed using Fisher's exact test. $\mathrm{P}<0.05$ was considered to indicate a statistically significant difference.

\section{Results}

Clinical characteristics. Among the 21 patients investigated, there were 9 cases of tumor metastasis. All patients demonstrated lymph node metastasis, and the average number ratio for neck lymph node metastasis (percentage of lymph node metastasis cases out of the total number of lymph node dissection) was $46.2 \%(312 / 675 \times 100=46.2 \%)$. A total of 15 patients underwent surgery once, 5 patients underwent surgery twice and 1 patient underwent surgery 6 times, prior to RAI. Pathological tumor-node-metastasis staging results were as follows: Stage I, 15 patients; stage III, 3 patients; and stage IVa, 3 patients. The interval between initial RAI and final surgery was $<3$ months in the majority of patients $(81.0 \% ; 17 / 21)$. All 21 patients were administered ${ }^{131} \mathrm{I}$ treatment $1-5$ times, and the average $\mathrm{Tg}$ level was $131.8 \mathrm{ng} / \mathrm{ml}$ (range, 0.2-500 ng/ml) pre-TSH stimulation. A total of 3 patients exhibited neck lymph node metastasis, confirmed by RxWBS together with local SPECT/CT imaging, and 4 patients exhibited mediastinal lymph node metastasis. There were $18 / 21$ patients who underwent RAI $<3$ times $(85.7 \%)$, and 2 patients who underwent RAI 4 times $(9.5 \%)$. The accumulated dosage for all patients was 3.7-30.34 GBq (mean, 17.39 GBq).

${ }^{131}$ I therapy effectiveness does not demonstrate statistical significance between $C R$ and $N R$. The following response rates were observed: CR, 23.8\% (5/21); PR, 71.4\% (15/21); and NR, $4.8 \%(1 / 21)$. The observed overall effectiveness was $95.2 \%(20 / 21)$. The difference in effectiveness was not statistically significant between the CR and NR groups for gender, age $\geq 45$ years (age at diagnosis), interval between initial RAI and final surgery, RAI frequency, presence or absence of extrathyroidal invasion and extrapulmonary metastasis (Table I).

Lung uptake and treatment effectiveness does not demonstrate statistical significance between diffuse and focused uptake patients. A total of 12 patients demonstrated diffuse uptake, and the remaining 9 exhibited focused uptake with decreased ${ }^{131}$ I deposition, with no CR. There were 5 cases of I-WBS negativity among the diffuse uptake patients, and 6 cases demonstrated reduced uptake compared with pre-treatment levels, with 1 patient demonstrating a progressive increase of $\mathrm{Tg}$ and $\mathrm{Tg}$ antibody levels during 5 treatments, and exhibiting diffuse nodules on chest CT (Fig. 1). The CR rate was $41.7 \%(5 / 12)$, and the difference in CR rate between the diffuse uptake and focused uptake patients was not statistically significant $(\mathrm{P}=0.123)$. 
Table I. Independent prognostic factors predicting radioiodine treatment efficacy in 21 differentiated thyroid cancer patients with computed tomography-negative pulmonary metastasis.

\begin{tabular}{|c|c|c|c|c|}
\hline Clinicopathological feature & Total patients, n (\%) & $\mathrm{CR}, \mathrm{n}$ & $\mathrm{PR} / \mathrm{NR}, \mathrm{n}$ & P-value \\
\hline Gender & & & & 0.450 \\
\hline Male & $11(52.4)$ & 2 & 9 & \\
\hline Female & $10(47.6)$ & 3 & 7 & \\
\hline Age at diagnosis, years & & & & 0.557 \\
\hline$<45$ & $14(66.7)$ & 3 & 11 & \\
\hline$\geq 45$ & $7(33.3)$ & 2 & 5 & \\
\hline Interval between intial RAI and final surgery, months & & & & 0.696 \\
\hline$\leq 3$ & $17(81.0)$ & 4 & 13 & \\
\hline$>3$ & $4(19.0)$ & 1 & 3 & \\
\hline Total RAI treatments & & & & 0.421 \\
\hline$\leq 3$ & $18(85.7)$ & 5 & 13 & \\
\hline$>3$ & $3(14.3)$ & 0 & 3 & \\
\hline Extrathyroidal invasion & & & & 0.353 \\
\hline Yes & $9(42.9)$ & 3 & 6 & \\
\hline No & $12(57.1)$ & 2 & 10 & \\
\hline Extrapulmonary metastasis & & & & 0.098 \\
\hline Yes & $7(33.3)$ & 0 & 7 & \\
\hline No & $14(66.7)$ & 5 & 9 & \\
\hline
\end{tabular}

RAI, radioiodine therapy; CR, complete response; PR, partial response; NR, no response.

A

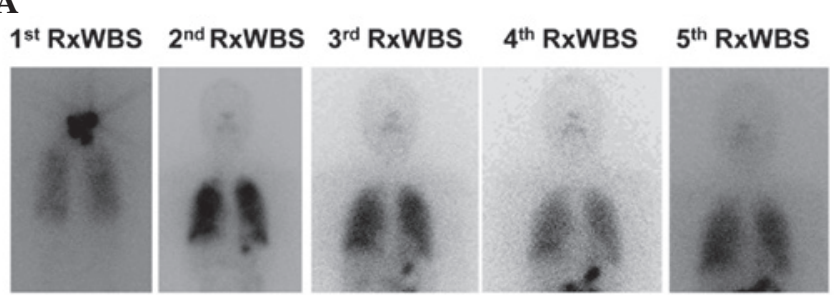

B

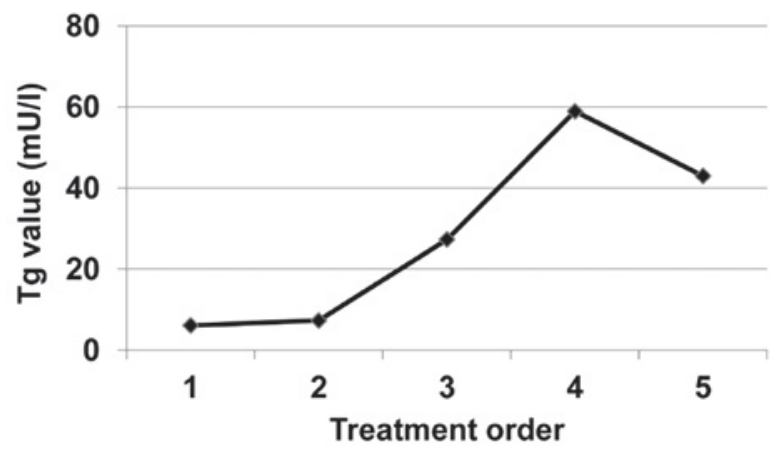

Figure 1. Chest CT images and Tg levels of a DTC patient exhibiting lung metastases during 5 RAI treatments. (A) Chest CT images of one DTC patient exhibiting lung metastases. (B) Serum Tg levels of the patient demonstrated progressive increase over the course of 5 RAI treatments. CT, computed tomography; Tg, thyroglobulin; DTC, differentiated thyroid cancer; RAI, radioiodine therapy; RxWBS, post-therapeutic whole-body scanning.

A correlation exists between $\mathrm{Tg}$ levels and extrapulmonary metastasis. All 21 patients were administered treatment 1-5 times, and 7 demonstrated extrapulmonary metastases in RxWBS. Among these 7 patients, 3 were identified to have neck lymph node metastases and 4 possessed mediastinal lymph node metastases. There was a correlation between $\mathrm{Tg}$ levels and extrapulmonary metastasis. All patients demonstrated extrapulmonary metastasis when $\mathrm{Tg}$ levels were $>87.5 \mathrm{ng} / \mathrm{ml}$ (area under curve, $1.0 ; \mathrm{P}<0.001$ ).

\section{Discussion}

The 10-year survival rate of PTC and FTC patients following 'thyroidectomy $+{ }^{131}$ Iodine radiotherapy + TSH inhibition' has been observed to be 93 and $85 \%$, respectively (9). However, almost $2 \%$ of PTC patients and $6 \%$ of FTC patients were diagnosed with distant metastases during their initial hospital visit or follow-up visit, and the survival rate is $<50 \%$ for these patients (10). The lungs are the most common metastatic sites for DTC, and the diagnosis is primarily dependent on medical imaging $(10,11)$. However, DTC lung metastases typically progress slowly and frequently present with a spotty lung distribution, various tumor sizes and tumor nodules with homogenous density $(12,13)$. Chest X-ray may frequently lead to an incorrect or missed diagnosis due to the detection limit (11). Relapsed DTC or metastatic DTC is frequently ${ }^{131} \mathrm{I}$-enriched. Therefore, ${ }^{131} \mathrm{I}-\mathrm{WBS}$ is one of the most specific methods for DTC diagnosis, not only for assessment of the therapeutic effect of ${ }^{131} \mathrm{I}$, but additionally for the diagnosis of DTC relapse or metastasis (4). In the present study, all patients underwent chest CT scanning prior to ${ }^{131} \mathrm{I}$ treatment, and 21 patients demonstrated no metastases, however, these patients were subsequently diagnosed with lung metastases during ${ }^{131}$ I radiotherapy. Thus, ${ }^{131}$ I therapy is more accurate for 
DTC staging, and contributes significantly to early diagnosis, early treatment and an improved prognosis.

The exact efficacy of ${ }^{131}$ I treatment for DTC lung metastases has not been consistent, and the response and CR rates vary widely among studies (range, 10-90\%) (6,14-16). In the present study, the response rate was $95.2 \%$, and the CR rate was $23.8 \%$, which were higher than the previously reported response rates. The reason underlying these superior response rates may be ascribed as due to several aspects. The survival time for ${ }^{131}$ I-treated DTC patients, including patients with lung metastases, is relatively long $(17,18)$. The criteria for specific studies may be different. Furthermore, although the ${ }^{131} \mathrm{I}$ treatment plan for DTC patients is relatively standard, the pre-treatment status of patients, the dosage choice, the follow-up design and the response standard may be different (12). In addition, current CT scanning is able to detect tumor nodules with diameters of 3-5 $\mathrm{mm}$. In patients with metastasis that is not detected by CT, the nodule size is likely to be $<3 \mathrm{~mm}$ in diameter and is possibly in the early stages of metastasis. However, the penetration distance of $\beta$-irradiation from ${ }^{131} \mathrm{I}$ inside the tissue is relatively short (average distance, $1 \mathrm{~mm}$ ) (18). For these patients, the lung deposits of ${ }^{131} \mathrm{I}$ are primarily located within the DTC tumor nodules, which allows the achievement of maximum local irradiation. This may explain why the ${ }^{131} \mathrm{I}$ treatment time and accumulated dosages were lower in the present study compared with previous studies, and is consistent with reports that the prognosis of patients exhibiting diffuse lung metastases is superior to that of patients with focused lung metastases, following ${ }^{131}$ I treatment (13).

Serum Tg is exclusively produced by the thyroid gland, and serum Tg will fall to markedly reduced levels following thyroidectomy or partial thyroidectomy and residual tissue removal with iodine radiotherapy. The return or increase of serum $\mathrm{Tg}$ levels indicates DTC treatment failure, reoccurrence or metastasis (6). Thus, serum $\mathrm{Tg}$ monitoring is a simple and reliable detection method for monitoring DTC reoccurrence, metastasis and therapy efficacy (14). In the present study, 20/21 patients demonstrated decreased serum $\mathrm{Tg}$ levels following ${ }^{131} \mathrm{I}$ therapy, and no new metastases were identified by follow-up CT scanning. American Thyroid Association guidelines have indicated that an increase in serum $\mathrm{Tg}$ levels $[>10 \mathrm{mg} / \mathrm{l}$ following the end of levothyroxine (LT4) administration] in the follow-up monitoring of DTC patients who received radioiodine therapy suggests metastasis, and ${ }^{131} \mathrm{I}$ therapy is recommended (3). However, there was no specific analysis concerning the significance of such an increase in Tg levels. In the present study, it was identified that $16 / 21$ patients demonstrated an increase in serum Tg levels $(>10 \mathrm{ng} / \mathrm{ml})$ in the initial RAI, following the conclusion of LT4 intake, and 7 patients (Tg levels, $>87.85 \mathrm{ng}$ / $\mathrm{ml}$ ) were confirmed to exhibit lung metastases in the subsequent RxWBS, with a statistically significant difference. Thus, it was suggested that DxWBS may not be essential for DTC patients demonstrating low serum Tg levels, but that it is necessary for patients exhibiting high levels of serum $\mathrm{Tg}$. This result requires additional investigation with a larger sample size. In the present study, no DxWBS was performed pre-RAI due to concerns regarding the 'stunning' effect, and it is possible that an insufficient RAI dosage was administered during the initial treatment. This hypothesis requires additional investigation with a larger sample size.
In conclusion, DTC patients exhibiting lung metastasis not detected by CT scanning responded well to ${ }^{131} \mathrm{I}$ radiotherapy and demonstrated a positive prognosis in the present study. Serum $\mathrm{Tg}$ levels prior to ${ }^{131} \mathrm{I}$ treatment may correlate with metastasis, and this may suggest the importance of diagnostic WBS prior to radiotherapy. This hypothesis requires additional investigation with a larger sample size.

\section{References}

1. Kim TY, Kim WG, Kim WB and Shong YK: Current status and future perspectives in differentiated thyroid cancer. Endocrinol Metab (Seoul) 29: 217-225, 2014.

2. Lin JD, Chao TC, Chou SC and Hsueh C: Papillary thyroid carcinomas with lung metastases. Thyroid 14: 1091-1096, 2004.

3. American Thyroid Association (ATA) Guidelines Taskforce on Thyroid Nodules and Differentiated Thyroid Cancer, Cooper DS, Doherty GM, Haugen BR, Kloos RT, et al: Revised American Thyroid Association management guidelines for patients with thyroid nodules and differentiated thyroid cancer. Thyroid 19: 1167-1214, 2009.

4. Ilgan S, Karacalioglu AO, Pabuscu Y, et al: Iodine-131 treatment and high-resolution CT: Results in patients with lung metastases from differentiated thyroid carcinoma. Eur J Nucl Med Mol Imaging 31: 825-830, 2004

5. Tachi Y, Iwano S, Kato K, Tadokoro $M$ and Naganawa S: Diagnostic whole-body scanning before radioiodine therapy for pulmonary metastases of differentiated thyroid cancer: Predictive value and recommendations. Clin Nucl Med 33: 845-851, 2008.

6. Phan HT, Jager PL, van der Wal JE, Sluiter WJ, Plukker JT, Dierckx RA, Wolffenbuttel BH and Links TP: The follow-up of patients with differentiated thyroid cancer and undetectable thyroglobulin ( $\mathrm{Tg}$ ) and $\mathrm{Tg}$ antibodies during ablation. Eur $\mathrm{J}$ Endocrinol 158: 77-83, 2008.

7. Pace L, Klain M, Albanese C, Salvatore B, Storto G, Soricelli A and Salvatore M: Short-term outcome of differentiated thyroid cancer patients receiving a second iodine-131 therapy on the basis of a detectable serum thyroglobulin level after initial treatment. Eur J Nucl Med Mol Imaging 33: 179-183, 2006.

8. Edge SB, Byrd DR, Compton CC, et al: AJCC Cancer Staging Manual. 7th edition. Springer-Verlag, New York, NY, USA, 2010.

9. Hundahl SA, Fleming ID, Fremgen AM and Menck HR: A National Cancer Data Base report on 53,856 cases of thyroid carcinoma treated in the U.S., 1985-1995 [see comments]. Cancer 83: 2638-2648, 1998.

10. Shaha AR, Shah JP and Loree TR: Differentiated thyroid cancer presenting initially with distant metastasis. Am J Surg 174: 474-476, 1997.

11. Bal CS, Kumar A, Chandra P, Dwivedi SN and Mukhopadhyaya S: Is chest X-ray or high-resolution computed tomography scan of the chest sufficient investigation to detect pulmonary metastasis in pediatric differentiated thyroid cancer? Thyroid 14: 217-225, 2004.

12. Lang BH, Wong KP, Cheung CY, Wan KY and Lo CY: Evaluating the prognostic factors associated with cancer-specific survival of differentiated thyroid carcinoma presenting with distant metastasis. Ann Surg Oncol 20: 1329-1335, 2013.

13. Casara D, Rubello D, Saladini G, et al: Different features of pulmonary metastases in differentiated thyroid cancer: Natural history and multivariate statistical analysis of prognostic variables. J Nucl Med 34: 1626-1631, 1993.

14. Ma C, Xie J and Kuang A: Is empiric 131I therapy justified for patients with positive thyroglobulin and negative 131I whole-body scanning results? J Nucl Med 46: 1164-1170, 2005.

15. Shaha AR, Shah JP and Loree TR: Low-risk differentiated thyroid cancer: The need for selective treatment. Ann Surg Oncol 4: 328-333, 1997

16. Pitoia F, Bueno F and Cross G: Long-term survival and low effective cumulative radioiodine doses to achieve remission in patients with 131Iodine-avid lung metastasis from differentiated thyroid cancer. Clin Nucl Med 39: 784-790, 2014.

17. Higashi T, Nishii R, Yamada S, et al: Delayed initial radioactive iodine therapy resulted in poor survival in patients with metastatic differentiated thyroid carcinoma: A retrospective statistical analysis of 198 cases. J Nucl Med 52: 683-689, 2011.

18. Grosev D, Loncarić S, Huić D and Dodig D: Geometric models in dosimetry of thyroid remnant mass. Nuklearmedizin 47 : $120-126,2008$ 